\title{
Aplicación inteligente en la práctica profesional del contador público en el área fiscal
}

\author{
Intelligent Application in the Professional Practice of the Public Accountant \\ in the Tax Area
}

Aplicação inteligente na prática profissional do contador público na área tributária

Alfonso Aldape Alamillo

Tecnológico Nacional de México, Instituto Tecnológico de Ciudad Juárez, México aaldape@itcj.edu.mx https://orcid.org/0000-0001-8921-9479

Juan Pedro Benítez Guadarrama Universidad Autónoma del Estado de México, México jpbenitezg@uaemex.mx https://orcid.org/0000-0002-2826-6359

Francisco Zorrilla Briones

Tecnológico Nacional de México, Instituto Tecnológico de Ciudad Juárez, México fzorrilla@itcj.edu.mx https://orcid.org/0000-0003-0553-9841 


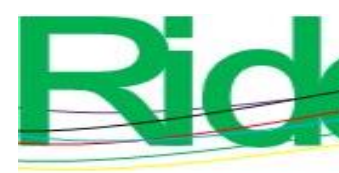

Revista Iberoamericana para la Investigación y el Desarrollo Educativo ISSN $2007-7467$

applied to 50 certified public accountants, who used the tool at the Colegio Profesional de Contadores Públicos in the Estado de México. The procedure for obtaining data was carried out in five stages (purpose, description, analysis, practice and evaluation). After its application, data processing was carried out using the SPSS version 20 program. By applying descriptive and inferential statistics, it was possible to know the quality level of the intelligent fiscal application and each of the variables that make it up: Functionality, Reliability, Usability, Efficiency, Maintainability and Portability. The results showed that specific functions and properties to meet user needs and effectively fulfill fiscal tasks or activities offer excellent performance.

Keywords: computer application, income tax, practical work, taxation.

\section{Resumo}

O objetivo deste estudo foi projetar e avaliar um aplicativo inteligente a ser utilizado pelo contador público como ferramenta na prestação de seus serviços profissionais. Com o objetivo de informar o contribuinte, principalmente as pessoas físicas com atividade empresarial, sobre os tributos federais no México, como o imposto de renda (ISR) e o imposto sobre o valor agregado (IVA). O design da aplicação inteligente foi estruturado de acordo com o disposto na legislação tributária do período 2019. Para a avaliação, foi criado um instrumento baseado na norma ISO 9126, norma internacional que especifica as variáveis que constituem a qualidade da aplicação. Este instrumento foi aplicado a 50 contadores públicos credenciados, que utilizaram a ferramenta na Associação Profissional de Contadores Públicos do Estado do México. O procedimento de obtenção dos dados foi realizado em cinco etapas (objetivo, descrição, análise, prática e avaliação). Após a sua aplicação, o processamento dos dados foi realizado no programa SPSS versão 20. Por meio da aplicação da estatística descritiva e inferencial, foi possível conhecer o nível de qualidade da aplicação fiscal inteligente e cada uma das variáveis que a compõem: Funcionalidade, Confiabilidade , Usabilidade, Eficiência, Capacidade de Manutenção e Portabilidade. Os resultados mostraram que funções e propriedades específicas para atender às necessidades do usuário e cumprir com eficácia as tarefas ou atividades fiscais oferecem excelente desempenho.

Palavras-chave: aplicação informática, imposto de renda, trabalho prático, tributação. 


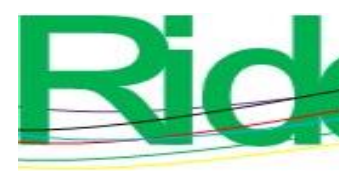

Fecha Recepción: Abril 2020
Revista Iberoamericana para la Investigación y el Desarrollo Educativo ISSN $2007-7467$

\section{Introducción}

La tecnología móvil ha traído nuevas formas de realizar actividades académicas y profesionales. Al mismo tiempo, dicha tecnología ha traído nuevas exigencias en uno y otro ámbito. Particularmente, el uso de medios electrónicos en las funciones y actividades del contador público se distingue por traer soluciones inmediatas a ciertas actividades relacionadas con el contexto fiscal. ¿Cuánto tengo que pagar?, ¿cómo se calcula el impuesto sobre la renta (ISR)?, ¿cómo se calcula el impuesto al valor agregado (IVA)?, ¿por qué tengo que pagar ese importe?, ¿qué puedo deducir?, ¿cómo tengo que realizar mis operaciones?, son algunos de los cuestionamientos que se suscitan constantemente en el área fiscal.

El objetivo de este estudio fue diseñar y evaluar una aplicación inteligente para ser utilizada por el contador público como herramienta en la prestación de sus servicios profesionales. Esto con el propósito de informar al contribuyente, en especial a las personas físicas con actividad empresarial, sobre los impuestos federales en México, tal y como el ISR y el IVA. El diseño de la aplicación inteligente se estructuró conforme a las disposiciones contenidas en las leyes fiscales del periodo 2019. Para la evaluación se creó un instrumento con base en la norma ISO 9126, norma internacional que especifica las variables que conforman la calidad de la aplicación. Dicho instrumento fue aplicado a 50 contadores públicos certificados, quienes utilizaron la herramienta en el Colegio Profesional de Contadores Públicos, en el Estado de México. El procedimiento para la obtención de datos se realizó en cinco etapas (propósito, descripción, análisis, práctica y evaluación). Después de su aplicación, se realizó el procesamiento de datos mediante el programa Statical Package for the Social Sciences (SPSS, versión 20). Al aplicar estadística descriptiva e inferencial, se pudo conocer el nivel de calidad de la aplicación inteligente fiscal y cada una de las variables que la conforman: Funcionalidad, Confiabilidad, Usabilidad, Eficiencia, Mantenibilidad y Portabilidad.

Los resultados arrojaron que las funciones y propiedades específicas para cubrir las necesidades del usuario y cumplir con eficacia las tareas o actividades fiscales ofrecen un rendimiento de nivel excelente. Al ser utilizado por el usuario en condiciones normales de operación, el tiempo de respuesta es igualmente satisfactorio. La facilidad de uso, de 


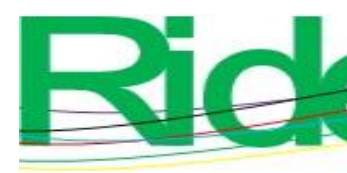

Revista lberoamericana para la Investigación y el Desarrollo Educativo ISSN 2007 - 7467

aprendizaje, de procesamiento rápido de datos, de modificar y corregir hacen que la herramienta inteligente muestre una seguridad en los resultados sobre los impuestos a cargo del contribuyente, y su portabilidad permite ser utilizada en cualquier momento que se requiera. La herramienta proporciona información confiable, comparable, comprensible y relevante, útil para el cumplimiento de las obligaciones fiscales de los contribuyentes mexicanos, todo lo cual va encaminado a evitar sanciones, infracciones y multas que afecten el patrimonio de la entidad o negocio y a mejorar los procesos de gestión tributaria; puede ser vista como una guía para presentar la declaración en la plataforma de la autoridad hacendaria. En suma, garantiza un desempeño profesional con calidad; proporciona información confiable, relevante y comprensible en el momento que se requiera por el contratante del servicio, y proporciona las bases para informar con certeza y precisión. Sin duda la aplicación puede ser utilizada e incorporada en las prácticas fiscales a fin de mejorar la gestión de la recaudación fiscal en México.

\section{Antecedentes del problema}

La Constitución Política de los Estados Unidos Mexicanos (2020), considerada como la ley suprema en México (Quintanilla y Rojas, 1999; Sánchez, 2014), establece la obligación de los mexicanos de contribuir a los gastos públicos de la federación, del estado y del municipio en que se residan. Del contenido de esta disposición emana la obligación del pago del impuesto por parte de los empresarios a partir de las disposiciones establecidas por una ley federal o estatal. Además, los impuestos deben ser equitativos y justos considerando la capacidad económica del sujeto de la relación tributaria.

A partir del ordenamiento constitucional, se origina un vínculo jurídico entre dos sujetos: el sujeto activo representado por el Estado, quien está facultado para exigir el cumplimiento y pago de los impuestos en México, y el sujeto pasivo, representado por el empresario, quien tiene la obligación de cumplir con las disposiciones establecidas en las leyes fiscales materializadas a través del pago de los impuestos. Teniendo en cuenta este binomio, Gianni (citado en Diez, 2005) establece que el Estado administra el patrimonio derivado de la recaudación de los tributos, y los invierte directamente en el desarrollo de sus actividades. Garza (2005), por su parte, refiere que el Estado y los demás entes públicos realizan acciones encaminadas a obtener ingresos necesarios para sostener los servicios públicos. 


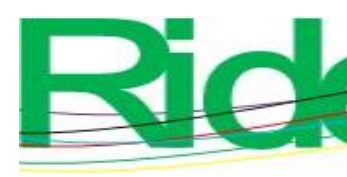

Revista Iberoamericana para la
Investigación y el Desarrollo Educativo ISSN 2007-7467

A esta actividad se le conoce como actividad financiera del Estado: regula los ingresos ordinarios y extraordinarios y los egresos de la organización estatal. Para cumplir con dicha actividad, el Estado, a través de la Secretaría de Hacienda y Crédito Público (SHCP) (organismo encargado de la recaudación de los impuestos), implementa estrategias que facilitan al contribuyente la gestión tributaria. Entre ellas, en las últimas décadas, ha establecido plataformas de gobierno electrónico o e-gobierno, las cuales permiten transitar del modelo tradicional de Estado burocrático a un modelo moderno de Estado proveedor de bienes y servicios (Carranza, 2002). El Gobierno mexicano debe aprovechar las tecnologías de la información y comunicación (TIC) para mejorar los procesos de gestión interna de la administración pública y así otorgar mejores servicios, facilitar la obtención de aplicaciones, permitir el acceso a la información, incrementar la rendición de cuentas, la transparencia y fortalecer la participación ciudadana (Secretaría de la Función Pública [SFP], 2019).

Una alternativa de mejora en los procesos de la gestión tributaria es la incorporación de tecnología flexible: el gobierno móvil (m-gobierno), que hace referencia a las TIC relacionadas con las tecnologías inalámbricas o móviles como los celulares/smartphones, computadoras portátiles y asistentes digitales personales (PDA, por sus siglas en inglés) conectados a redes locales inalámbricas (Carrión y Larenas, 2009, p. 8).

El smartphone o teléfono inteligente es un dispositivo de comunicación que puede ser trasladado de un lugar a otro. Este dispositivo permite la comunicación de voz, imágenes, texto, videos y sonidos; permite el entretenimiento con juegos de diferentes plataformas, el acceso a Internet y múltiples funciones de utilidad práctica que impactan en la vida social, laboral y profesional de sus usuarios (Malo, Casas, Figuer y González, 2006).

En una nota publicada en El Economista (Notimex, 28 de junio de 2019) se reporta que durante el primer trimestre de 2019 se contabilizaron en el país 120.7 millones de líneas móviles, $3.6 \%$ más que la base alcanzada el año anterior. De ellas, $81.8 \%$ o 98.7 millones corresponden a la modalidad de prepago y 22.0 millones $(18.2 \%)$ a pospago, de acuerdo con un estudio de la consultora The Competitive Intelligence Unit (The CIU). ¿Cuántos de ellos se utilizan para conectarse al m-gobierno? No hay respuesta. Sin embargo, todo buen profesional de la contabilidad, que tiene clientes que requieren cubrir obligaciones hacendarias, hace uso de tecnologías inalámbricas para eficaz y eficientemente cumplirle al cliente. 


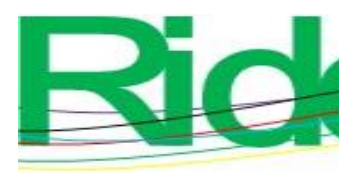

Revista Iberoamericana para la Investigación y el Desarrollo Educativo ISSN $2007-7467$

El m-gobierno ha sido una herramienta útil para los contadores, sin embargo, cada año tienen que resolver un problema: los cambios fiscales que anualmente son instrumentados por el Gobierno federal. Estos cambios originan la necesidad de que el contador reciba capacitación para entender los cambios, el porqué de estos y cómo aplicarlos. Acuden a seminarios, conferencias, a las asociaciones y colegios de contadores y a instituciones de educación superior en busca del conocimiento pertinente.

Quienes acuden a actualizaciones constantes de la profesión contable y a capacitaciones a través de cursos presenciales y a distancia hacen uso de las TIC. Esta situación es la base de la idea que sustenta el estudio aquí presentado: desarrollar y evaluar una aplicación para teléfono inteligente que lleve al usuario a prestar un servicio profesional de una forma ágil y eficaz, y al mismo tiempo, facilitar su trabajo.

La complejidad de las leyes hacendarias mexicanas, las reformas que anualmente se les hacen, la ausencia de eficiencia y eficacia en el asesoramiento contable al empresario afectan al momento de cumplir, en tiempo y forma, con las obligaciones legales hacendarias de la persona física o del dueño del negocio.

Los contadores requieren medios digitales que simplifiquen los procedimientos para cumplir con las exigencias del Servicio de Administración Tributaria (SAT). El uso del teléfono inteligente está evolucionando en la práctica fiscal; está promoviendo el diseño y desarrollo de aplicaciones fiscales con base en las leyes hacendarias vigentes. Por ello, el propósito fundamental, base del estudio aquí presentado, es tener una aplicación inteligente que contribuya al profesionista o contribuyente a mejorar el proceso y a realizar el pago y entero de los impuestos federales. La utilización de la aplicación electrónica no solo favorecerá al contador público, sino al contribuyente en el pago de sus impuestos en tiempo y forma y a la autoridad para lograr incrementar la recaudación fiscal en México.

\section{Método}

En este apartado se exponen los pasos que se llevaron a cabo en el desarrollo del estudio, sin perder nunca de vista el objetivo de la investigación: vincular el entorno actual con la práctica que realiza el profesionista contador a fin de facilitar su desenvolvimiento en el ámbito fiscal. 


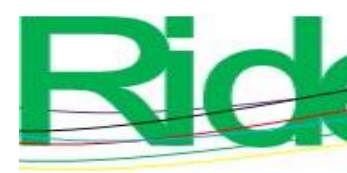

Revista lberoamericana para la Investigación y el Desarrollo Educativo ISSN 2007-7467

El diseño de la aplicación inteligente se realizó teniendo en cuenta los conceptos contenidos en la Ley del Impuesto Sobre la Renta (Cámara de Diputados del H. Congreso de la Unión, 11 de diciembre de 2013) y la Ley del Impuesto al Valor Agregado (Cámara de Diputados del H. Congreso de la Unión, 29 de diciembre de 1978) y el desarrollo de las aplicaciones incorporadas por el Gobierno federal para el proceso del pago y entero del tributo federal.

En el diseño se consideraron todos los elementos necesarios para mejorar las condiciones en la determinación y cálculo de los impuestos federales. La metodología normativa fue igualmente considerada a fin de cumplir con un servicio de calidad: informar, presentar y promover el cumplimiento de las obligaciones fiscales del contribuyente.

\section{Descripción del método}

1) El diseño y desarrollo de la aplicación móvil se llevó a cabo cuidando cumplir con las disposiciones fiscales vigentes mexicanas, y así lograr determinar y calcular los impuestos federales. Esto mediante la programación extrema, también conocida como metodología XP, la cual es una metodología ágil y flexible utilizada para el desarrollo de software. Se utilizó, también, el programa Crimson Editor para desarrollar el lenguaje de programación Java y la plataforma Java 2 Micro Edition, orientada al desarrollo de aplicaciones para dispositivos pequeños con capacidades limitadas, como el caso del teléfono celular, en el que es posible aplicar el archivo ejecutable para realizar la práctica fiscal (Prieto, 2005).

2) Con el propósito de ejemplificar la aplicación del teléfono celular en la práctica fiscal, se consideraron los datos de un contribuyente del mes de enero del 2019.

3) Manualmente se llevó a cabo el procedimiento del cálculo del ISR del mes de enero 2019: se calculó la utilidad o ganancia, restando del total de los ingresos obtenidos en tal mes las deducciones autorizadas (los gastos del mismo periodo); al resultado obtenido, se le aplicó la tabla del artículo 96 de la Ley del Impuesto sobre la Renta (Cámara de Diputados del H. Congreso de la Unión, 11 de diciembre de 2013) correspondiente, y a la cantidad determinada se le restaron las retenciones que fueron efectuadas por personas morales.

4) El cálculo del IVA se realizó restando al total del IVA cobrado o IVA trasladado (originado de las ventas) el total del IVA retenido por la persona moral y el IVA pagado (IVA acreditable) originado de los gastos del mes. Aquí podemos tener dos posibles 


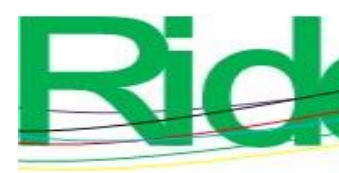

Revista Iberoamericana para la Investigación y el Desarrollo Educativo ISSN 2007 - 7467

resultados: IVA a pagar o IVA a favor. El IVA a pagar es cuando el IVA cobrado es mayor a la sumatoria del IVA retenido y el IVA pagado; cuando el IVA cobrado más el retenido es mayor al IVA pagado tendremos un resultado de IVA a favor, que puede ser disminuido por el IVA a pagar de periodos posteriores.

5) Los resultados obtenidos en la herramienta fiscal deben descargarse en el formato electrónico proporcionado por la autoridad fiscal, el cual deberá presentarse en la institución autorizada más cercana al contribuyente o en la plataforma electrónica del banco.

6) Para evaluar la calidad de la aplicación fiscal, el instrumento se aplicó a 50 contadores públicos que se encuentran actualmente dedicados a prestar servicios profesionales independientes de asesoría/consultorías a personas físicas. Todos los participantes están asociados con los conceptos que se manejaron y los botones contenidos para calcular el ISR e IVA.

\section{Implementación del método}

Pasando a la operacionalización, el método se dividió en cinco fases.

\section{Primera fase}

Al inicio del proceso de la evaluación se les explicó a los participantes el procedimiento general y se les solicitó su cooperación voluntaria para utilizar en una situación real y luego evaluar la aplicación inteligente fiscal mediante un instrumento diseñado para tal propósito.

a) Se les explicó lo que es una aplicación fiscal.

b) Además, se les especificó el tipo de tecnología en el que se puede utilizar la aplicación fiscal. Asimismo, se puntualizó que el desarrollo del modelo tecnológico se elaboró con base en la legislación fiscal mexicana.

c) Posteriormente, se proporcionó el archivo por medio del correo electrónico, bluetooth y WhatsApp para ser cargado en su teléfono personal, y así utilizar la aplicación fiscal e iniciar el proceso de evaluación. 


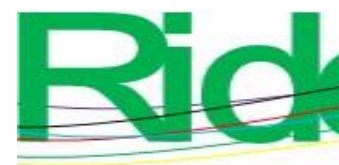

Revista Iberoamericana para la Investigación y el Desarrollo Educativo ISSN 2007 - 7467

\section{Segunda fase}

En esta fase se describieron los conceptos que integran la aplicación fiscal en cada uno de los impuestos que intervienen en la determinación.

a) Se determinaron y explicaron los montos que deben ingresar para obtener los resultados requeridos por la autoridad hacendaria.

\section{Tercera fase}

En la tercera fase se aplicó el cuestionario de 20 ítems (anexo 1), el cual fue piloteado, previamente, con una muestra de cinco participantes, profesionistas prestadores de servicios activos en la base de datos del SAT, quienes señalaron que las preguntas no presentaban dificultades para su comprensión, por lo que se procedió a su aplicación.

\section{Cuarta fase}

En la cuarta fase se llevó a cabo una sesión por grupos con una duración de 50 minutos aproximadamente, donde se presentó el software, se explicó su funcionamiento, se realizaron casos prácticos reales de contribuyentes dedicados a prestar servicios profesionales independientes en un municipio del Estado de México; a continuación, se les proporcionaron los datos de un contribuyente para utilizar la aplicación fiscal; de esta forma se capacitó a los sujetos para usar el software.

\section{Quinta fase}

Se llevó a cabo el procesamiento y análisis de los datos. Para ello, se utilizó el paquete estadístico SPSS (versión 20), programa estadístico informático usado como instrumento de análisis cuantitativo que facilita el manejo de los datos obtenidos en la investigación de campo. Mediante este programa se realizó lo siguiente:

a) Un análisis descriptivo, con el propósito de tener una visión general sobre los resultados obtenidos.

b) Un análisis inferencial, con el propósito de argumentar sobre las afirmaciones de los participantes y con el objetivo de encontrar el grado de correlación existente entre las variables de la calidad; además, se usó la regresión lineal para predecir las variables que influyen con mayor fuerza en la calidad. 


\section{Resultados}

La muestra fue elegida de una población de 50 sujetos; $100 \%(n=50)$ aceptó voluntariamente participar en el estudio. En cuestiones de género, $60 \%(n=30)$ eran hombres y $40 \%(n=20)$ mujeres; todos emplearon la aplicación fiscal en su celular como herramienta para obtener la información fiscal sobre los impuestos federales (tabla 1).

Tabla 1. Distribución de la muestra por género

\begin{tabular}{|l|c|c|}
\hline Género & Frecuencia & Porcentaje \\
\hline Femenino & 20 & 40 \\
\hline Masculino & 30 & 60 \\
\hline Total & 50 & 100.0 \\
\hline
\end{tabular}

Fuente: Elaboración propia

Por lo que respecta a la edad de los participantes, $18.0 \%(n=9)$ tenía 32 años, $18.0 \%$ $(n=9)$ tenía 33 años, $18.0 \%(n=9)$ tenía 34 años, $18.0 \%(n=9)$ tenía 35 años, $18.0 \%$ $(n=9)$ tenían 37 años y $10.0 \%(n=5) 36$ años (tabla 2).

Tabla 2. Distribución de la muestra por edad

\begin{tabular}{|l|c|c|}
\hline Edad & Frecuencia & Porcentaje \\
\hline 32 años & 9 & $18.0 \%$ \\
\hline 33 años & 9 & $18.0 \%$ \\
\hline 34 años & 9 & $18.0 \%$ \\
\hline 35 años & 9 & $18.0 \%$ \\
\hline 36 años & 5 & $10.0 \%$ \\
\hline 37 años & 9 & $18.0 \%$ \\
\hline Total & 50 & $100.0 \%$ \\
\hline
\end{tabular}

Fuente: Elaboración propia

Respecto al tipo de servicios que prestaban los participantes, $34 \%(n=17)$ era de comercio, $28.0 \%(n=14)$ era de servicios y $38 \%(n=19)$ era de transformación (tabla 3). 
Tabla 3. Distribución por prestación de servicios

\begin{tabular}{|l|c|c|}
\hline Servicios & Frecuencia & Porcentaje \\
\hline Comercio & 17 & $34.0 \%$ \\
\hline Servicios & 14 & $28.0 \%$ \\
\hline Transformación & 19 & $38.0 \%$ \\
\hline Total & 50 & $100.0 \%$ \\
\hline
\end{tabular}

Fuente: Elaboración propia

En la tabla 4 se muestra la media $(\mu)$ y desviación estándar $(\sigma)$ de los factores predictores de la calidad, a saber: Funcionalidad, Fiabilidad, Usabilidad, Eficiencia, Mantenibilidad y Portabilidad; como se puede apreciar, el nivel tiende a ser excelente.

Tabla 4. Factores predictores de la calidad

\begin{tabular}{|c|c|c|c|c|c|c|c|}
\hline & \multicolumn{6}{|c|}{ Factores } & \multirow{2}{*}{ Tota } \\
\hline & 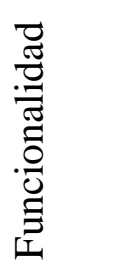 & 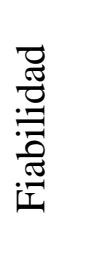 & 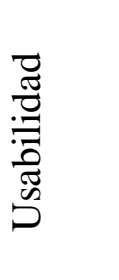 & 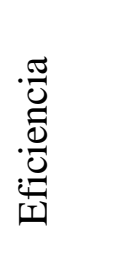 & 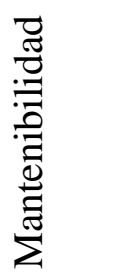 & 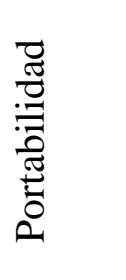 & \\
\hline \multirow[t]{2}{*}{$\mu$} & & & & & & & \\
\hline & 2.20 & 2.70 & 1.60 & 1.50 & 2.90 & 2.04 & 2.16 \\
\hline$\Sigma$ & 0.981 & 0.641 & 0.320 & 0.207 & 0.220 & 0.180 & 0.424 \\
\hline
\end{tabular}

Fuente: Elaboración propia

Respecto de la relación entre las subescalas, así como de la calidad, podemos observar que existe una correlación muy fuerte entre los factores: Usabilidad, Funcionalidad, Eficiencia y Mantenibilidad (tabla 5). 
Tabla 5. Correlación de Pearson

\begin{tabular}{|c|c|c|c|c|c|c|c|}
\hline \multicolumn{8}{|c|}{ Variables de calidad } \\
\hline & 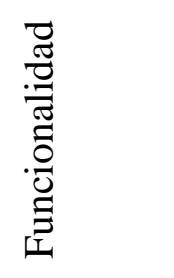 & 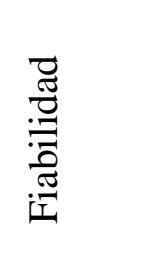 & 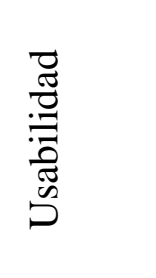 & 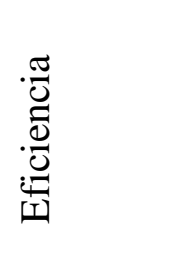 & 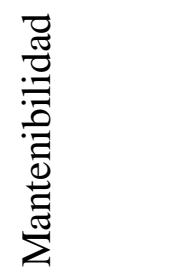 & 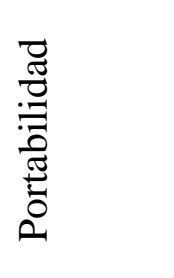 & 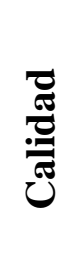 \\
\hline Funcionalidad & 1.0 & & & & & & \\
\hline Fiabilidad & $0.414 * *$ & 1.0 & & & & & \\
\hline Usabilidad & $0.748^{* *}$ & $0.755^{* *}$ & 1.0 & & & & \\
\hline Eficiencia & $0.506^{* *}$ & $0.678^{* *}$ & $0.827 * *$ & 1.0 & & & \\
\hline Mantenibilidad & $0.853^{* *}$ & $0.473 * *$ & $0.724 * *$ & $0.457 * *$ & 1.0 & & \\
\hline Portabilidad & $0.494^{* *}$ & $0.222 * *$ & $0.701^{* *}$ & $0.585 * *$ & $0.411 * *$ & 1.0 & \\
\hline Calidad & $0.845^{* *}$ & $0.675^{* *}$ & $0.970 * *$ & $0.805^{* *}$ & $0.826^{* *}$ & $0.732 * *$ & 1.0 \\
\hline
\end{tabular}

$* p<0.05 ; * * p<0.01$

Fuente: Elaboración propia

\section{Discusión}

La muestra estuvo compuesta por $40 \%$ de mujeres y $60 \%$ de hombres. En este estudio existe una ligera variación entre los participantes, lo cual refleja una oportunidad de equilibrio tecnológico entre géneros.

Las edades oscilaron entre los 32 a los 37 años. El dato más representativo indica que $73.7 \%$ pertenece al rango de 32 a 34 años. Los participantes de mediana edad tienen mayor habilidad en el manejo de la tecnología, por tanto, tienden a la maximización de los recursos tecnológicos, lo que genera confiabilidad y certeza en la evaluación del instrumento.

Asimismo, $100 \%$ de los participantes está involucrado en la prestación de servicios profesionales; de manera desglosada, $34 \%$ en actividades de comercio, $28 \%$ en servicios y $38 \%$ dedicado a la transformación de artículos o productos, lo cual enriquece y contribuye al cumplimiento del objetivo de la investigación, además de proporcionar información certera sobre los resultados de la evaluación. Las aplicaciones tecnológicas favorecen y potencializan la labor de los contadores, ya que están enfocadas en los requerimientos de 


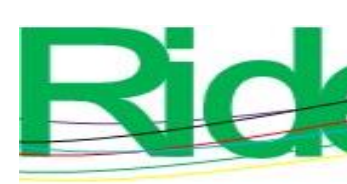

Revista Iberoamericana para la
Investigación y el Desarrollo Educativo ISSN 2007-7467

cada actividad, por tanto, posibilitan la correcta y oportuna presentación de información fiscal ante la autoridad pertinente.

El instrumento de medición obtuvo un coeficiente alfa de Cronbach de $\propto=0.921$, lo que sugiere un nivel de confiabilidad excelente.

La percepción de los sujetos en general muestra un alto cumplimiento respecto a los factores de Fiabilidad ( $\mu=2.7000$ y una desviación estándar de 0.64138), Eficiencia $(\mu=1.5000$ con desviación estándar de 0.207) y Funcionalidad $(\mu=2.2000$ con una desviación estándar de 0.98143). En estas variables, la menor desviación -estándar corresponde a Fiabilidad. Las variables de Portabilidad $(\mu=2.0467$ con desviación estándar de 0.1800 ), Usabilidad ( $\mu=1.6000$ con desviación estándar de 0.3200) y Mantenibilidad de la herramienta $(\mu=2.9000$ con desviación estándar de 2.220) presentan una tendencia buena de cumplimiento en los criterios de calidad, además de especificar una desviación estándar casi uniforme en la opinión de los participantes.

En la correlación de Pearson se encontraron 21 correlaciones significativas de un total de 21 posibles. Las correlaciones más altas se dieron entre Funcionalidad y Mantenibilidad $(r=0.853)$. La calidad muestra correlaciones positivas significativas con Usabilidad $(r=0.970)$, Funcionalidad $(r=0.845)$, Mantenibilidad $(r=0.826)$, Eficiencia $(r=0.805)$, Portabilidad ( $r=.0732$ ) y Fiabilidad ( $r=0.414)$; no hubo variables con la que no presentaran una relación significativa con la calidad de la aplicación fiscal.

Los coeficientes de determinación $\left(r^{2}\right)$ permitieron conocer el nivel en que cada variable independiente permite predecir el comportamiento de la dependiente. Los resultados sugieren que el comportamiento de las variables Funcionalidad, Confiabilidad, Usabilidad, Eficiencia, Portabilidad y Mantenibilidad predicen en $94.0 \%$ el comportamiento de la variable de calidad de la aplicación fiscal.

La variable con muy alto nivel en la predicción del comportamiento de la calidad es Usabilidad $\left(r^{2}=0.940\right)$; con un nivel alto de predicción son Funcionalidad $\left(r^{2}=0.712\right)$, Mantenibilidad $\left(r^{2}=0.681\right)$ y Eficiencia $\left(r^{2}=0.647\right)$; con un moderado nivel de predicción, Portabilidad $\left(r^{2}=0.536\right)$ y la variable Confiabilidad $\left(r^{2}=0.456\right)$. Estadísticamente, todas muestran un nivel de predicción significativo de la variable dependiente. 


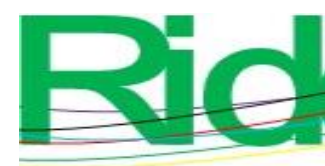

Revista Iberoamericana para la Investigación y el Desarrollo Educativo ISSN 2007 - 7467

\section{Conclusiones}

La app, como herramienta de solución de casos prácticos en la actividad fiscal, cuenta con niveles de calidad excelentes; a partir de ello se puede inferir que garantiza un desempeño profesional óptimo, y proporciona información confiable, relevante y comprensible para complementar tareas fiscales.

Cuando se incorporan las TIC, además de resolver de forma más inmediata y práctica los casos, también el profesional contador tiene la posibilidad de desarrollar paulatinamente habilidades, aptitudes y actitudes intercontextuales; en suma, adquirir de forma autónoma nuevas competencias tendientes al desarrollo personal y profesional en el contexto fiscal. El uso de las apps especializadas en el área fiscal con calidad no solo promueve las competencias, además se crea una nueva forma de solución a los problemas suscitados en un contexto fiscal de manera inmediata; se presenta un modelo en el cual se pueden generar diferentes escenarios de simulación real que permite al profesionista la generación de información útil para el empresario y así este pueda optimizar los recursos empresariales.

El uso de las aplicaciones móviles especializadas que garanticen la funcionalidad en dispositivos inteligentes equipos electrónicos, la portabilidad, esto es, ser transportada de un lugar a otro, la mantenibilidad, permanecer ejecutada cuando se requiera, la confiabilidad de la información que presenta, la usabilidad que se le da para los fines que se persiguen, la eficiencia en cuanto a la optimización del recurso electrónico en el procesamiento de datos, permite que el usuario obtenga información financiera, comprensible, confiable, relevante y comparable para cumplir con las obligaciones legales y contribuir con desarrollo empresarial y obtener rendimientos sobre los recursos invertidos en la entidad 


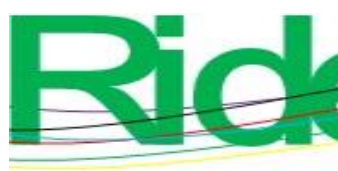

Revista Iberoamericana para la Investigación y el Desarrollo Educativo ISSN 2007 - 7467

\section{Referencias}

Cámara de Diputados del H. Congreso de la Unión. (29 de diciembre de 1978). Ley del Impuesto al Valor. Diario Oficial de la Federación. Recuperado de http://www.diputados.gob.mx/LeyesBiblio/pdf/77_091219.pdf.

Cámara de Diputados del H. Congreso de la Unión. ( 11 de diciembre de 2013). Ley del Impuesto sobre la Renta. Diario Oficial de la Federación. Recuperado de http://www.diputados.gob.mx/LeyesBiblio/pdf/LISR_091219.pdf.

Carranza, J. (2002). E-fiscal en América Latina: experiencias líderes en sistemas de información de administración financiera, compras del gobierno y administración tributaria. Ponencia presentada en el VII Congreso Internacional del CLAD sobre la Reforma del Estado y de la Administración Pública. Lisboa, del 8 al 11 de octubre de 2002.

Recuperado

de http://unpan1.un.org/intradoc/groups/public/documents/CLAD/clad0043821.pdf.

Carrión, H., Larenas, R. y Carrión, M. (2009) Gobierno móvil. Conceptos, posibilidades de la aplicación y una experiencia en el Ecuador. Quito, Ecuador: Grupo Faro. Recuperado de http://www.imaginar.org/docs/L_mgobierno_NED.pdf.

Constitución Política de los Estados Unidos Mexicanos. (2020). México: Edición BOB.

Diez, R. (2005). Análisis constitucional de la ley del impuesto al activo. (tesis de licenciatura). Universidad de las Américas Puebla, Cholula.

Garza, S. (2005). Derecho financiero mexicano (26. ${ }^{a}$ ed.). Edición México: Porrúa.

Malo, S., Casas, F., Figuer, C. y González, M. (2006). El teléfono móvil: disponibilidad, usos y relaciones por parte de los adolescentes entre 12 y 16 años. Estudios sobre Educación, (10), 55-78

Notimex. (28 de junio de 2019). México cerró marzo de 2019 con 120.7 millones de líneas celulares; $81.8 \%$ son de prepago. El Economista. Recuperado de https://www.eleconomista.com.mx/empresas/Mexico-cerro-marzo-de-2019-con120.7-millones-de-lineas-celulares-81.8-son-de-prepago-20190628-0055.html.

Prieto, M. (2005). Desarrollo de juegos con J2ME: Java Micro Edition. México: Alfaomega. Quintanilla, J. y Rojas, J. (1999). Derecho tributario mexicano (4a . ed). México: Trillas.

Sánchez, J. (2014). Nociones de derecho fiscal. México: Publicaciones Administrativas y Contables Jurídicas. 


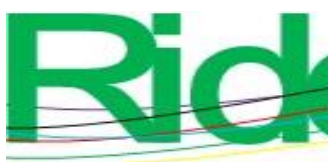

Revista Iberoamericana para la Investigación y el Desarrollo Educativo ISSN $2007-7467$

Secretaría de la Función Pública [SFP]. (2019). Unidad de Gobierno Digital. México: Secretaría de la Función Pública. Recuperado de http://pcop.funcionpublica.gob.mx/index.php/ua/ssfp/ugd.html.

\section{Anexo 1 \\ Calidad sobre la app para el pago de impuestos federales de las personas físicas}

El objetivo de este instrumento es tener su opinión acerca de la calidad que tiene la aplicación (app) para el pago provisional del régimen profesional.

Se le solicita sea objetivo en sus respuestas, por tal motivo, lea cuidadosamente.

Instrucciones: Marque con un $x$ en el número que considere, sea lo más objetivo posible.

- ¿Cuál es su edad?:

- ¿ ¿Cuál es su género?: (M) (F)

- $\quad$ Número de veces que usas el celular al día: (1 a 25) (26 a 50) (51 a 100)

- Para que usas con más frecuencia el celular: (Comunicación) (Entretenimiento) (Redes sociales) (Juegos) (Internet)

- $\quad$ Has descargado aplicaciones: (Gratis) (Pago)

- $\quad$ Cuentas con internet en tu móvil: (Sí) (No)

- $\quad$ Marca de celular: (Huawei) (LG) (Samsung) (Nokia)

- $\quad$ Prestación de servicios: (Comerciales) (Industriales) (Servicios)

$$
1=\text { Malo } \quad 2=\text { Deficiente } \quad 3=\text { Regular } \quad 4=\text { Bueno } \quad 5=\text { Excelente }
$$




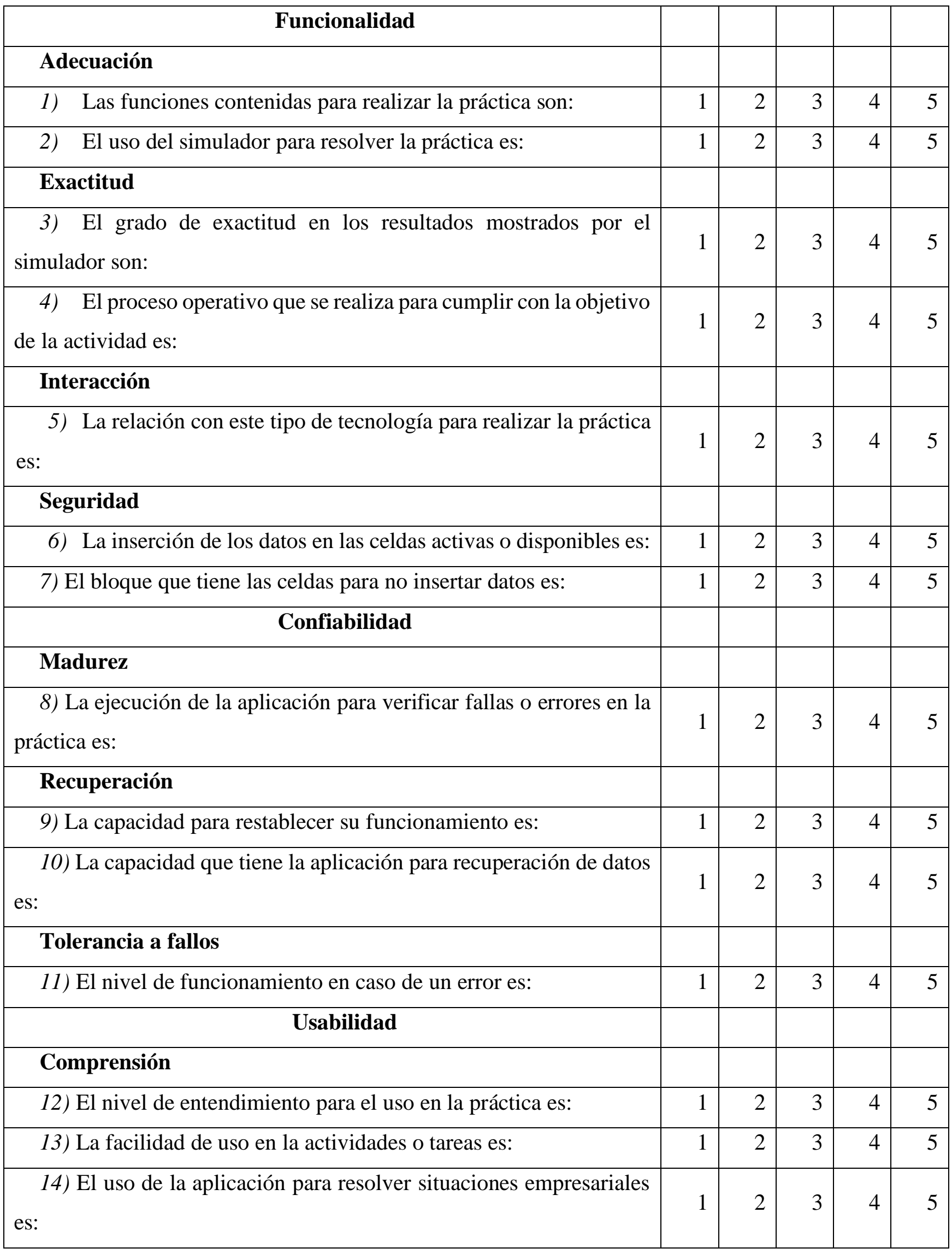




\begin{tabular}{|c|c|c|c|c|c|}
\hline Aprendizaje & & & & & \\
\hline 15) El nivel de aprendizaje para su uso en la práctica es: & 1 & 2 & 3 & 4 & 5 \\
\hline \multicolumn{6}{|l|}{ Operatividad } \\
\hline 16) El nivel de operación en la práctica es: & 1 & 2 & 3 & 4 & 5 \\
\hline 17) El nivel de esfuerzo para su uso en la práctica es: & 1 & 2 & 3 & 4 & 5 \\
\hline \multicolumn{6}{|l|}{ Atracción } \\
\hline 18) Las características que presenta para su uso en la práctica es: & 1 & 2 & 3 & 4 & 5 \\
\hline \multicolumn{6}{|l|}{ Eficiencia } \\
\hline \multicolumn{6}{|l|}{ Tiempo } \\
\hline 19) El tiempo de respuesta en los resultados es: & 1 & 2 & 3 & 4 & 5 \\
\hline 20) El tiempo del procesamiento de la información es: & 1 & 2 & 3 & 4 & 5 \\
\hline 21) Los beneficios obtenidos en la realización de las funciones son: & 1 & 2 & 3 & 4 & 5 \\
\hline \multicolumn{6}{|l|}{ Recurso } \\
\hline $\begin{array}{l}\text { 22) La capacidad que tiene el teléfono inteligente como herramienta } \\
\text { para la práctica es: }\end{array}$ & 1 & 2 & 3 & 4 & 5 \\
\hline $\begin{array}{l}\text { 23) La utilización de la app en el teléfono inteligente sin conexión } \\
\text { a internet es: }\end{array}$ & 1 & 2 & 3 & 4 & 5 \\
\hline \multicolumn{6}{|l|}{ Mantenibilidad } \\
\hline \multicolumn{6}{|l|}{ Análisis } \\
\hline $\begin{array}{l}\text { 24) La identificación de cada uno de los elementos que la integran } \\
\text { es: }\end{array}$ & 1 & 2 & 3 & 4 & 5 \\
\hline 25) El diagnóstico realizado con la ayuda de la herramienta es: & 1 & 2 & 3 & 4 & 5 \\
\hline \multicolumn{6}{|l|}{ Cambios } \\
\hline 26) Las modificaciones realizadas en la herramienta son: & 1 & 2 & 3 & 4 & 5 \\
\hline \multicolumn{6}{|l|}{ Estabilidad } \\
\hline $\begin{array}{l}\text { 27) La funcionalidad de las herramientas ante diversas } \\
\text { modificaciones es: }\end{array}$ & 1 & 2 & 3 & 4 & 5 \\
\hline \multicolumn{6}{|l|}{ Prueba } \\
\hline $\begin{array}{l}\text { 28) Las pruebas realizadas en la herramienta para la solución de la } \\
\text { práctica es: }\end{array}$ & 1 & 2 & 3 & 4 & 5 \\
\hline Portabilidad & & & & & \\
\hline
\end{tabular}




\begin{tabular}{|l|r|r|r|r|r|}
\hline Adaptabilidad & & & & & \\
\hline 29) La aceptación de la herramienta en la práctica es: & 1 & 2 & 3 & 4 & 5 \\
\hline Instalación & & & & & \\
\hline 30) La facilidad de instalar la herramienta es: & 1 & 2 & 3 & 4 & 5 \\
\hline Coexistencia & & & & & \\
\hline 31) La funcionalidad en las tabletas electrónicas es: & 1 & 2 & 3 & 4 & 5 \\
\hline 32) La funcionalidad en emuladores Android es: & 1 & 2 & 3 & 4 & 5 \\
\hline 33) La funcionalidad en teléfonos inteligentes es: & 1 & 2 & 3 & 4 & 5 \\
\hline Reemplazamiento & & & & & \\
\hline 34) La facilidad de reemplazo de la aplicación es: & 1 & 2 & 3 & 4 & 5 \\
\hline
\end{tabular}

¡Gracias por su tiempo!

Observaciones:

Sugerencias: 